\title{
Aspectos éticos de la investigación en clases de ciencias: dilemas resueltos y no resueltos
}

Kenneth Tobin
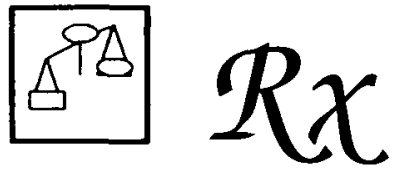

En los últimos años el aula está convirtiéndose en uno de los escenarios favoritos de la investigación educativa. Con ello aumentan los problemas éticos y sociales y el peligro de que el educador se convierta, premeditadamente o no, en presa y víctima del investigador-depredador.

En este articulo se aborda este problema y se aportan propuestas sencillas y concretas para resolverlo de modo que la investigación contribuya a fertilizar y no a confundir el ambiente educativo.

\section{INTRODUCCION}

Es seguro afirmar que que, a medida que los tipos de investigación han evolucionado, también lo han hecho los interrogantes que rodean la práctica de la investigación en las aulas. No hace mucho, era inusual encontrar investigadores que realmente se adentraran en las aulas y examinaran las vidas de enseñantes y estudiantes. La investigación sobre la enseñanza y el aprendizaje era más bien una experiencia relativamente indirecta donde las ventanas abiertas a las aulas eran las respuestas dadas a elementos de instrumentos basados en lápiz y papel. Después asistimos a una era de instrumentos de observación en la que observadores experimentados codificaban lo que ocurría en categorías preestablecidas. A medida que la etnografía iba tomando posición en la investigación educativa, los estudios se hacían más largos y los investigadores empezaron a explorar y a encontrar significados desde el punto de vista de los participantes. Por tanto, la naturaleza de realizar investigaciones en las aulas cambió drásticamente en un periodo relativamente breve y, en conjunción con estos cambios, la naturaleza de las prácticas éticas (es decir, prácticas consideradas como correctas o morales) también ha cambiado.

La conducta moral se inscribe en un contexto que, en sí mismo, es un componente de la cultura. En consecuencia, las cuestiones éticas deberían ser consideradas dentro de un contexto cultural. Desde el punto de vista del investigador, es necesario mostrar una conducta moral hacia todos los participantes en una cultura. Las oportunidades para llevar a cabo conductas no éticas abundan en la investigación en las aulas y en otros tipos de investigación en las escuelas. Desde el momento en que un equipo de investigación empieza a negociar su entrada en una cultura (es decir, en una escuela, un distrito escolar, etc.), es necesario tener precaución para garantizar que los intereses de 
los participantes en la cultura no sean perjudicados por su participación en el estudio. Estos intereses no están protegidos por una adherencia ritual a normas establecidas antes de un estudio sino ejerciendo un extremo cuidado en todo momento, manteniendo los intereses de los participantes en un primer plano mental. Como consecuencia, las cuestiones que se deben abordar desde un punto de vista ético variarán en el transcurso de un estudio concreto y de un estudio al siguiente. No es que las consideraciones éticas tengan más importancia en los estudios de un tipo que en los de otro (por ejemplo, más en los estudios cualitativos que en los cuantitativos); sin embargo, cuando los investigadores realmente visitan la sede de un estudio, tienen oportunidades para mantener un mayor contacto personal con los participantes y aumenta el potencial para actuar de una manera que vaya en perjuicio de los intereses de los participantes.

Ser un investigador ético requiere una cuidadosa consideración de dos culturas: en primer lugar (y más importante) la cultura a la que pertenecen los participantes en la investigación (frecuentemente la cultura de las escuelas), y en segundo lugar la cultura de los investigadores con sus costumbres, creencias y prácticas. Entre estas creencias son importantes las que se refieren a la naturaleza de la investigación y los objetivos de publicación. Por ejemplo, mi código de conducta moral desde la perspectiva de un investigador destaca la responsabilidad de llevar a cabo una investigación con el fin de aprender y de comunicar lo que aprenda a otros investigadores, a los enseñantes y a la administración.

En muchos aspectos, este estudio es un viaje personal a través de más de 15 años de investigación en las aulas. El tema principal lo forman las cuestiones éticas que se han ido planteando, el contexto en el que la ética ha constituido una preocupación y la manera de abordar los problemas. Un tema que impregna todo el artículo es que las cuestiones éticas dependen del contexto y, en consecuencia, si el contexto cambia también cambian las cuestiones que pueden considerarse de carácter ético.

\section{Asignación a un grupo de control}

La ética de llevar a cabo investigaciones en las aulas se convirtió para mí en una cuestión de preocupación consciente y personal durante un estudio del tiempo de servicio y el rendimiento en ciencias (Tobin y Capie, 1982). Al final de este estudio apareció un problema. Una de las enseñantes se enfadó cuando supo que su clase había estado en el grupo de control y no había rendido tan bien como las clases de un grupo experimental. Al ser una enseñante escrupulosa, no consideró que la necesidad de validar la estrategia de tiempo de servicio fuera razón suficiente para que sus alumnos fueran perjudicados por no haber utilizado un tiempo de servicio extendido durante el estudio. No estaba convencida de las virtudes de realizar investigaciones para validar prácticas educativas. Para ella, bastaba con que pudiera ver el sentido de adoptar una estrategia como el tiempo de servicio para su práctica docente. Tampoco le importaba mucho el bien superior que se pudiera alcanzar mediante el estudio.

Mis actividades como investigador no eran éticas desde la perspectiva de la enseñante porque negué a sus alumnos la oportunidad de aprender tanto como pudieran. Desde mi punto de vista, yo había llevado a cabo el estudio con integridad y había seguido las normas de la profesión investigadora. Sin embargo, la enseñante no tuvo oportunidad de elegir si quería estar incluida en el grupo experimental, y tampoco sus alumnos tuvieron esta oportunidad. Ofrecer un tratamiento al grupo de control no fue una respuesta satisfactoria a las cuestiones planteadas por esta enseñante. En el núcleo de su actitud se encontraba la cuestión de tener el derecho y la responsabilidad profesional para tomar decisiones educativas para su clase y de no tener un conocimiento pleno de los detalles del estudio. Si esta enseñante hubiera sabido que estaba en un grupo de control es probable que hubiera declinado participar. Y si los estudiantes hubieran sabido que iban a estar en un grupo de control, es de suponer que algunos de ellos también habrían optado por no participar. 
La investigación en la que intervienen grupos experimentales y de control, ¿está predestinada a carecer de ética? Hablando retrospectivamente, es fácil ver lo que se podría haber heho para evitar el problema descrito anteriormente. Sin embargo, mi principal preocupación residía en la validez interna de un cuasi-experimento bastante complejo. El estudio implicaba más de un tratamiento y todos los enseñantes recibán atención y feedback sobre lo que se observaba que ocurría. Las quejas de la enseñante antes mencionada fueron una sorpresa y emanaron de mi decisión de compartir los resultados del estudio con los participantes en él.

Si no le hubiera comentado a la enseñante las relaciones entre el tiempo de servicio y el rendimiento en ciencias, es muy probable que hubiera estado satisfecha con los recursos ofrecidos a su clase por participar en el estudio y el feedback exhaustivo que se le ofrecía sobre su enseñanza. Su clase parecía beneficiarse mucho del hecho de participar en el estudio. Sin embargo, desde mi punto de vista como investigador, el curso de acción más ético fue proporcionar a todos los enseñantes implicados un informe detallado de lo que habíamos aprendido del estudio. El problema mencionado podría haberse evitado si los enseñantes hubieran sido informados de que el estudio implicaba grupos de control y que la asignación de los grupos se determinaría al azar.

\section{Compartir notas de campo e informes con los participantes}

La subjetividad de las observaciones en la investigación en las aulas se convirtió en objeto de estudio en un trabajo que se centraba en la enseñanza de un único ensenante ejemplar de ciencias (Tobin et al., 1988). En este estudio, un equipo de cuatro investigadores examinó la enseñanza y el aprendizaje en todas las clases impartidas por el enseñante durante un período cercano a los tres meses. Como era usual en anteriores estudios interpretativos, ofrecimos al enseñante nuestras notas de campo cada día. Los alumnos también podían leer estas notas de campo si se mostraban interesados en ello. Por tanto, los participantes podían leer lo que estábamos observando para que no se sintieran amenazados por todas las páginas escritas. A intervalos regulares durante el estudio ofrecíamos al enseñante informes escritos pero con la precaución de no sensibilizarlo respecto a las interpretaciones más destacadas para nosotros. En las conclusiones del estudio ofrecimos al enseñante un informe completo de lo que habíamos aprendido a partir del estudio. ¡El enseñante se puso furioso con lo que leyó!

En respuesta a nuestro informe de 30 páginas mecanografiadas a doble espacio, él elaboró una respuesta que aún era más larga. El informe que nosotros habíamos considerado objetivo e imparcial era percibido como subjetivo y parcial. A sus ojos, nuestra interpretación parecía implicar que era un enseñante ineficaz y ponía en duda nuestro derecho a hacer tales juicios e inferencias. Esta actitud del enseñante puso de relieve el abismo que existía entre las perspectivas del equipo de investigación y las del propio enseñante en clase. Aunque algunos miembros de nuestro equipo habían enseñado en escuelas incluso el año anterior, el enseñante asociaba sus ideas a una torre de marfil. El enseñante creía que no éramos ni realistas ni prácticos y que no nos enfrentábamos a las realidades de la vida en las aulas. Nuestras ideas teóricas sobre cómo aprenden los estudiantes no tenían lugar en la vida de ese enseñante, que sentía sobre él el impacto de fuerzas que le exigían preparar a los estudiantes para la universidad, cubrir los contenidos del texto, satisfacer las demandas de pruebas de alcance estatal y de niveles mínimos, y cumplir con las expectativas de padres y administradores. Estos dos marcos de referencia opuestos (es decir, el del enseñante y el de los investigadores) no se podían reconciliar y decidimos incorporar. al estudio tanto los puntos de vista del enseñante como los del equipo. El enseñante estuvo de acuerdo en que el informe era justo, pero no cedió ni un ápice en los puntos de desacuerdo. Podíamos apreciar la racionalidad de los argumentos del enseñan- 
te, pero disentíamos de sus premisas básicas. Este estudio, más que cualquier otro, me hizo reflexionar sobre el diseño de los estudios sobre enseñanza y aprendizaje. Decidí evitar traumas como estos en investigaciones futuras manteniendo al enseñante plenamente informado de nuestras interpretaciones a lo largo de todo el estudio.

Sin embargo, mantener al enseñante informado no era la cuestión ética de este estudio. Al enseñante se le mantenía informado, pero él simplemente estuvo en desacuerdo con las interpretaciones del equipo de investigación, y creyó que los roles del enseñante en las clases de ciencias debían ser distintos de los valorados por los miembros del equipo de investigación. La cuestión ética del estudio es que el enseñante se sintió herido por la experiencia. Su amor propio quedó rebajado por la experiencia de participar en el estudio. Se le dieron todas las notas de campo y los informes para que los leyera y su voz quedó representada en el informe del estudio, que no fue enviado para su publicación hasta que él acordó que era justo y dio su permiso para publicar el informe. Además, dimos pasos para garantizar que su identidad real fuera difícil de establecer.

En este ejemplo, se planteó una cuestión ética porque seguimos reglas establecidas antes del estudio. Habíamos acordado la regla de compartir todos los materiales escritos con el enseñante y, visto retrospectivamente, este curso de acción no fue adecuado. El estudio destaca las dificultades que tiene ofrecer a los enseñantes análisis de su actividad docente. Cuando un investigador o un equipo de investigadores elige centrarse en un rol determinado del enseñante e ignorar otros, existe el problema potencial de que el enseñante haya otorgado una prioridad baja al rol en el que se han centrado los investigadores. Ofrecer a los enseñantes una descripción de la enseñanza que destaque algo a lo que ellos no dan mucho valor es invitarles a tener una reacción negativa o, como mínimo, a tener diferencias de opinión.

Aunque se tenga cuidado para garantizar que los análisis sean imparciales, las diferencias en los marcos de referencia interpretativos del enseñante y del observador conducen a una situación en la que ciertos acontecimientos valorados se destacan y otros no. En consecuencia, se desarrolla un sesgo en lo que pudieran parecer observaciones objetivas de la enseñanza y el aprendizaje. Nuestros estudios sugieren que los enseñantes definen sus roles de maneras que les permitan tener éxito. El énfasis dado a los roles desempeñados por un enseñante depende de la manera en que se conceptulizan estos roles y de las creencias y los valores asociados a ellos. Por tanto, plantear preguntas sobre lo que un enseñante lleva a cabo en clase es plantear preguntas sobre sus creencias, sus valores y sus conceptualizaciones de los roles. Lo importante es que plantear estas preguntas puede perjudicar el amor propio y la profesionalidad del enseñante. En tales circunstancias, no se deberían plantear preguntas sin dedicar mucha consideración y sensibilidad a los sentimientos y las necesidades del enseñante. Una consideración importante es la preparación del enseñante para enfrentarse a preguntas que podrían dar como resultado una disminución de su amor propio.

El aspecto clave que no supimos reconocer es que ni las observaciones ni las interpretaciones son objetivas. Cuando un equipo de investigación visita aulas, ocurren demasiadas cosas y es imposible anotar ni siquiera la mayor parte de lo que acontece. Lo que se observa y luego se anota depende del objetivo de la investigación y de las creencias, conocimientos y valores (es decir, de los esquemas mentales) de los miembros del equipo de investigación. En la medida en que enseñantes e investigadores tengan diferentes perspectivas y esquemas mentales, cabe prever que se produzcan desacuerdos sobre la pertinencia y la representatividad de lo que se registra en las notas de campo. Cuando estos mismos datos se interpretan, cabe esperar que las discrepancias sean aún mayores. A pesar de este fallo en reconocer la importancia de los esquemas mentales en la observación y la interpretación de experiencias, hemos aprendido mucho de estos estudios cualitativos en las aulas. Además, en todos los casos el conocimiento obtenido de estos estudios se podría utilizar casi inmediatamente en programas de mejora para enseñantes. Independientemente de 
estos aspectos positivos de los estudios, yo me sentía inquieto por las cuestiones éticas que quedaban sin resolver asociadas a una disminución del amor propio de los participantes en investigaciones desarrolladas en la clase.

\section{Investigar en colaboración con los enseñantes}

La investigación en colaboración con los enseñantes implica varios supuestos previos. En primer lugar, y más importante, el diseño presupone que los enseñantes tienen amplios conocimientos prácticos que pueden estar o no disponibles en forma verbal. Estos conocimientos permiten a los enseñantes realizar unas aportaciones singulares a las reuniones del equipo de investigación. Un papel importante de los restantes miembros del equipo es ayudar a los enseñantes a verbalizar conocimientos profesionales pertinentes con el fin de contribuir al programa de la investigación. En segundo lugar, los enseñantes pueden aportar perspectivas singulares sobre la enseñanza y el aprendizaje. En tercer lugar, es probable que la información recibida por el enseñante se pueda utilizar para modificar lo que ocurre en el aula lo más pronto posible después de haber recibido la información. En consecuencia, se espera que las prácticas educativas cambien constantemente y que los entornos de aprendizaje asociados también lo hagan.

El diseño de estudios en los que el enseñante es un investigador pueden hacerle intervenir en la planificación de la investigación, en la recopilación, el análisis y la interpretación de los datos, y en la comunicación de los resultados. El enseñante no solo participa en la identificación del problema a investigar sino que también interviene en el diseño de estrategias para la recopilación de datos y en la interpretación de los datos recopilados. Por último, el enseñante interviene en la decisión sobre lo que se va a comunicar y la manera en que se redactará. Como coautor, el enseñante conoce con todo detalle lo que se va a comunicar y participa en la formulación y la edición de informes de lo que se ha aprendido.

Sin embargo, los problemas asociados con las dificultades que los enseñantes puedan tener en sus clases no desaparecen simplemente porque el enseñante sea ahora un investigador. Los enseñantes tienen problemas y no siempre los enmarcan de la misma manera que sus colegas del equipo de investigación. Esta cuestión puede ser causa de conflictos y requiere mucha consideración. Cuando un investigador identifica un problema en una clase, se trata del problema de otra persona. El enseñante-investigador, que para empezar puede que no haya percibido el problema, se enfrenta de repente a un aspecto que él no ha planteado y ve que otras personas esperan de él que haga cambios al respecto. Aunque exista un acuerdo sobre los cambios concretos que se deben realizar, puede darse un desacuerdo sobre si estos cambios se producen o no en la dirección deseada. Distintos esquemas mentales pueden conducir a perspectivas muy diferentes sobre lo que ocurre, sobre los datos que se recopilan y sobre la formulación de las preguntas.

La investigación con los enseñantes como miembros del equipo de investigación es muy exigente y hasta agotadora. Para que el equipo de investigación sea productivo, hace falta una gran cantidad de respeto y confianza entre los miembros del equipo. Debe intentarse comprender los puntos de vista de todos los miembros del equipo de investigación y, en la medida de lo posible, retener las perspectivas individuales en las fases de diseño, recopilación, interpretación y comunicación de un estudio. Sin embargo, cuando examinamos los conocimientos, las creencias y los valores del enseñante-investigador, también estamos examinando su vida privada y las cuestiones éticas abundan. Algunas de ellas se ilustran en el siguiente análisis de un estudio sobre ciencias de segunda enseñanza llevado a cabo por un equipo de tres personas: una enseñante-investigadora llamada Marsha, un estudiante graduado y yo mismo (Tobin y Ulerick, 1989). 
A primera vista, Marsha debería haber sido una eficaz enseñante de ciencias. Tenia unos buenos antecedentes en enseñanza de ciencias y había impartido cursos con éxito en el nivel universitario. Pero las cosas no salieron como estaba previsto. El entorno de la escuela hacía que todo enseñante nuevo fuera puesto a prueba por los alumnos. Marsha impartía dos clases por día: ciencias físicas y ciencias terrestres. Cada lección era observada y el equipo de investigación se reunía varias veces por semana para recopilar, analizar e interpretar datos. Marsha experimentó importantes dificultades con sus dos clases. Los estudiantes la sometieron a prueba y pronto vieron que no podía arreglárselas. Durante un corto período de tiempo los estudiantes fueron incontrolables. En consecuencia, Marsha era incapaz de centrarse en potenciar el aprendizaje de sus alumnos y tampoco estaba mentalizada para ello. Se dedicó a sobrevivir. Los alumnos eran antipáticos con ella, y ella les pagaba con la misma moneda.

Para los investigadores, las reuniones del equipo eran muy tristes. Mientras intentábamos encontrar una razón de la falta de éxito en las aulas de Marsha sondeando sus valores, sus creencias y sus conocimientos, relacionábamos lo que observamos en las aulas con lo que Marsha decía creer. En muchas ocasiones había largos silencios y era difícil discutir lo que ocurría porque pocas cosas funcionaban como estaba previsto y la enseñante-investigadora se encontraba bajo una gran tensión en las reuniones del equipo.

Las entrevistas eran difíciles de realizar. El método abierto empleado identificaba una cuestión planteando un problema y luego dejaba que Marsha estructurara su respuesta como quisiera. Nuestra estrategia consistía en pedirle que relacionara lo que ella creía (o decía que creía) con lo que se percibía que ocurría en clase. Al principio del estudio se puso de manifiesto que Marsha estaba emocionalmente deshecha porque en clase estaba haciendo cosas que no eran coherentes con sus creencias.

Marsha estaba abierta a propuestas para mejorar su enseñanza. Decidimos que hacían falta medidas fuertes para restablecer el sistema de control. Tras horas de discusiones sobre normas, expectativas y aplicación de las normas, Marsha y la dirección de su departamento implementaron el curso de acción que nosotros habíamos formulado. De un día para otro se produjeron mejoras drásticas. En vez de tener que centrarse en el control, Marsha podía dedicarse a su rol de facilitadora del aprendizaje. Durante un período de varias semanas, pudo introducir trabajos en grupo, actividades prácticas, el empleo de mapas de conceptos y, en general, hacer participar a los estudiantes de una manera activa.

Tener a Marsha en el equipo de investigación fue extremadamente beneficioso. Nos facilitó datos que no se podían obtener razonablemente de un enseñante que no se sintiera plenamente comprometido con el estudio como un colega de confianza. Por ejemplo, Marsha realizó dos borradores de un resumen de diez páginas mecanografiadas sobre sus creencias asociadas a la evaluación. La recopilación de estos datos exigió una gran cantidad de tiempo y no hay duda de que aprendimos cosas que no podríamos haber aprendido con cualquier otro diseño. Por ejemplo, aprendimos sobre el trauma asociado al cambio. Si no hubiéramos estado con Marsha al interpretar los datos, no hubiera sido posible estudiar la reflexión con tanto detalle. Gracias a ello pudimos tener el privilegio de observar la angustia que comportaba tener que volver a conceptualizar los roles de la enseñanza y descartar creencias que eran la base de actividades sin éxito en el contexto en el que enseñaba Marsha.

¿Cuáles eran las principales cuestiones éticas de este estudio? La cuestión que ponderábamos constantemente era si debíamos o no hacer lo que estábamos haciendo. ¿Era razonable seguir reuniéndonos día tras día con Marsha cuando discutir su falta de éxito implicaba tanta tensión? Había ocasiones en las que todos dudábamos de si debíamos continuar con el estudio. Sin embargo, la decisión siempre estuvo en manos de Marsha. Ella sabía que podía finalizar el estudio en cualquier momento.

De nuestros estudios con Marsha aprendimos mucho sobre cuestiones de ética. Empezamos el estudio con el punto de vista, algo ingenuo, de que en el estudio se 
minimizarían los juicios. Sin embargo, este estudio iba a versar sobre el cambio: un estudio que iba a implicar reflexión sobre la acción. Pero por su propia naturaleza, el estudio se proyectó para la formulación de juicios. Entonces, ¿quién tenía que emitir los juicios? Lo ideal hubiera sido que Marsha hubiera emitido los juicios y tomado las decisiones. Sin embargo, de ser ese el curso de acción elegido, los cambios que se necesitaban nunca se hubieran producido. Marsha necesitaba que alguien centrara sus reflexiones y esa persona tenía que emitir juicios. Participar en sesiones de reflexión con Marsha requería escuchar con sensibilidad. Aunque muchas de las cosas que dijo no eran objeto de comentarios, algunas de sus explicaciones revelaron creencias y metáforas que eran muy reveladoras de la manera en que concebía la enseñanza e implementaba el currículo. Estas explicaciones seleccionadas se convirtieron en foco de preguntas y posteriores discusiones. En ocasiones, era necesario sondear a Marsha e incomodarla cuando se yuxtaponían creencias conflictivas con el fin de que pudiera reflexionar sobre lo que ella creía. Este proceso llegó a ser tenso cuando Marsha se dio cuenta de que alguna de sus creencias más arraigadas y que habían dirigido sus acciones en clase ya no podían considerarse correctas. Tal como pretendía el estudio, el proceso de reflexión que se producía originó cambios y, en ocasiones, las discusiones fueron dolorosas y traumáticas para todos los miembros del equipo de investigación. Unas veces éramos conscientes de los juicios que se emitían durante las reuniones del equipo, y otras veces no.

Los conceptos importantes que aparecieron en relación a los juicios emitidos durante las reuniones del equipo se referían a la confianza, la sensibilidad a los sentimientos ajenos y el respeto mutuo fuera y dentro de las reuniones del equipo. Durante el estudio, se alcanzó un elevado nivel de confianza entre Marsha y los restantes miembros del equipo. $\mathrm{Y}$ durante las preguntas y explicaciones que se dieron en las reuniones del equipo, éramos sensibles al nivel de comodidad de Marsha. Eramos conscientes de la necesidad de frenarnos en ocasiones, durante una explicación o al plantear prguntas. Cuando las reuniones del equipo se centraban en el cambio tratamos de dejar que Marsha fuera quien más hablara y de limitar la longitud de nuestras propias explicaciones. Teníamos una idea de lo que Marsha podría conseguir y compartíamos una visión de la magnitud de los cambios necesarios en su clase y de la escala temporal en la que deberían producirse estos cambios.

\section{Epistemología}

Desde 1984, mi propio programa de investigación ha reflejado cada vez más la influencia de una epistemología constructivista radical (por ejemplo, von Glasersfeld, 1987). Es decir, se considera que el conocimiento es una construcción personal $\mathrm{y}$, aunque la realidad externa existe, los seres humanos no tienen acceso directo a ella. La objetividad no es posible puesto que el único acceso que tienen los seres humanos al mundo externo es a través de sus sentidos que, naturalmente, están conectados a sus cuerpos. Por tanto, la experiencia es una construcción personal y el aprendizaje se puede definir como el proceso social de comprender la experiencia en términos de lo que ya se conoce. Las implicaciones de llevar a cabo investigaciones que sigan una epistemología constructivista son sustanciales. Por ejemplo, ¿en qué consisten los datos de un estudio? Cuando empezamos a realizar investigaciones cualitativas en 1984 (por ejemplo, Tobin y Gallagher, 1987), considerábamos que los datos eran los contenidos de nuestros cuadernos de campo, las transcripciones de entrevistas, etc. Sin embargo, desde una perspectiva constructivista, es evidente que toda experiencia es histórica y que la diferencia importante entre los datos procedentes de notas de campo o de diarios personales y pensamientos reconstruidos, es el intervalo temporal entre la experiencia inicial y el acto de reconstruir el acontecimiento en forma de notas de campo, anotaciones en un diario o pensamientos. Empezamos a darnos cuenta de que cualquier pensamiento reconstruido podría considerarse un dato. Esta 
toma de conciencia es subversiva en muchos aspectos y nos hizo reconsiderar algunas de las posturas respecto a la ética que habían sido defendidas por enseñantes de ciencias que participaban en investigaciones en clase. Por ejemplo, no es sensato asignar la propiedad de los datos de un estudio a los enseñantes participantes. Después de todo, los datos son construcciones subjetivas de los individuos. Los datos no son objetivos y no se pueden transferir intactos desde la mente de un investigador a la mente de un enseñante. Entonces, ¿cómo se puede decir que los enseñantes son propietarios de los datos de un estudio? ¿Y en qué medida se puede impedir a los investigadores que utilicen los datos de un estudio?

Se plantean otras cuestiones éticas cuando la investigación se contempla desde una perspectiva constructivista. Consideremos la situación a la que se enfrentan los investigadores que examinan el proceso del cambio en un enseñante. ¿Es ético pretender cambiar la enseñanza? Si los enseñantes tienen unos fundamentos para lo que hacen y lo que hacen tienen sentido para ellos, ¿es defendible que un investigador juzgue que lo que hacen es inadecuado? Hemos encontrado que los enseñantes no están dispuestos a realizar cambios (por ejemplo, adoptar un enfoque al aprendizaje centrado en los problemas; Wheatley, 1991) o que no es probable que los mantengan si los cambios que han introducido no tienen sentido para ellos y no se percibe que sean mejores que lo que hacían antes de una intervención. Naturalmente, para que los cambios propuestos tengan sentido, normalmente es necesario interpretar el aprendizaje y la enseñanza desde una perspectiva constructivista. En nuestra experiencia, (por ejemplo, Tobin y Jakubowski, 1990) muchos enseñantes (y quizá la mayoría de ellos) adoptan una epistemología positivista para comprender lo que hacen. Esto les va bien ya que las prácticas tradicionales parecen estar basadas en el positivismo (Grundy, 1987). ¿Cuál es la justificación para que los investigadores esperen que los enseñantes modifiquen sus epistemologías y, a partir de ahí, que cambien lo que hacen en clase? Y si se intenta convencer a los enseñantes de las ventajas de realizar cambios, ¿se considera que estos intentos son moralmente correctos? Naturalmente, hemos respondido afirmativamente a la última pregunta. Creemos que es ético intentar mejorar la calidad de la enseñanza y el aprendizaje y que es responsabilidad de los enseñantes hacer lo que puedan para facilitar la mejora educativa. Lo que se considera como una mejora es un juicio de valor basado en la experiencia. Desde nuestra perspectiva, el constructivismo es el mejor modelo disponible para explicar el conocimiento y el aprendizaje y, como consecuencia, enseñar y aprender se pueden comprender en términos de constructivismo mejor que en términos de modelos distintos como el positivismo. Por tanto, nos sentimos cómodos ofreciendo a los enseñantes entornos que les permitan aprender sobre el constructivismo y comprender lo que hacen y lo que planean hacer. Si los enseñantes se muestran reticentes a cambiar o se niegan a hacerlo, no sería ético que el investigador les obligara a cambiar o a actuar de una manera que perjudique el amor propio de los participantes.

\section{Enfoques tradicionales a las cuestiones éticas}

Desde una perspectiva constructivista, los dilemas éticos no se pueden considerar independientemente de la cultura en la que se hallan inscritos. Un dilema se conceptualiza como un problema, como un obstáculo que se encuentra un investigador durante un estudio. Por tanto, se produce un dilema ético cuando un investigador se encuentra con una situación en la que no está seguro de cómo actuar para proteger y fomentar los intereses de todos los participantes en un estudio. La dificultad aumenta por la disponibilidad de patrones distintos a la hora de decidir cómo actuar de una manera ética (por ejemplo, perspectivas utilitarias, deontológicas y contractuales). 
Los enfoques utilitarios a la ética fomentan una mejora de los intereses de la mayoría de los participantes en una cultura. El utilitarismo impone una concepción del bien conjunto a expensas del bien de los menos favorecidos. Cuando los intereses de los individuos son contrarios a los intereses de la cultura, generalmente un investigador actuaría en pro de los intereses de la cultura y no a favor de los intereses individuales. El utilitarismo solo contempla las acciones, no lo que ocurre con el papel o la identidad moral del actor (Sichel, 1988, p. 67). En consecuencia, el utilitarismo es una forma de ética conductista en el sentido de que lo que ocurre dentro del cuerpo del actor -cómo cambia el yo moral- es relativamente poco importante. Solo las consecuencias externas de las acciones pueden satisfacer los principios del utilitarismo. Bernstein (1983, p. 157) observa que el empleo de una perspectiva utilitarista tiene sentido en una comunidad en la que se dé una aceptación de principios y normas de carácter ético. Un problema de la perspectiva utilitarista es que tiene el potencial de perpetuar las diferencias de poder existentes. Por tanto, en un contexto de investigación en clase, el enseñante, que tradicionalmente posee un estatus carente de poder en relación al investigador, puede permanecer en ese estado carente de poder como resultado de las acciones de un investigador basado en el utilitarismo.

Otro enfoque tradicional a los dilemas éticos es la deontología. Jeremy Bentham acuñó el término 'deontología' a partir de una palabra griega que significa 'lo que es adecuado' o 'algo que debiera ser'. Los juicios deontológicos, que dan respuesta a la cuestión de qué es lo correcto, normalmente proceden de normas o principios orientados a proteger los derechos de los individuos. La perspectiva utilitarista utiliza como referencia el principio de que una persona debe actuar como desea que actúen los demás en la misma situación. Esta perspectiva, que distancia al individuo de la decisión sobre cómo actuar, es objetivista porque desvincula un dilema moral de la vida concreta y las características personales de los implicados. En ausencia de una manera consensuada de actuar en situaciones determinadas, la perspectiva deontológica ofrece normas como referentes para las acciones. La ética deontológica se basa en la aplicación de normas desarrolladas por una comunidad, como una organización de investigadores en enseñanza de la ciencia, para especificar reglas aceptables de conducta en situaciones determinadas. Sin embargo, Sichel (1988) afirmaba que hacía falta algo más que una base deontológica para tomar decisiones de carácter moral. Afirmaba que, además de cumplir con un código de conducta, era necesario que una persona sopesara la situación e hiciera uso de su 'carácter' para determinar lo que era adecuado y lo que no lo era. Kohlberg y Candee adoptaron un punto de vista similar al discutir las decisiones de carácter ético. Observaron que un juicio de responsabilidad puede considerarse una afirmación de segundo orden de la voluntad de actuar de acuerdo con un juicio deontológico. La pregunta a responder no es solo por qué una acción es correcta sino también por qué un individuo debe actuar de una manera determinada. La función de un juicio de responsabilidad es determinar hasta qué punto lo que es moralmente bueno o correcto también es estrictamente necesario (Kohlberg y Candee, 1984, p. 518). Otros autores, como Noddings (1984), afirman que características personales como la preocupación por los demás ofrecen un base para la conducta moral. Sin embargo, Bell (1989) llegaba a la conclusión de que, por sí mismas, ni una ética deontológica ni una ética de preocupación pueden resolver el problema del relativismo moral (es decir, lo que se considera moralmente correcto varía de una persona a otra y de una cultura a otra). Según Bell:

\footnotetext{
... cualquier conflicto moral genuino, cuando es llevado al nivel de la reflexión, conduce a una reevaluación de la concepción del bien que tiene uno o de cómo se manifiesta en un contexto determinado, una reevaluación que no se aborda adecuadamente ni mediante el compromiso personal ni con la justicia (p. 107).
}

Con frecuencia se aboga por un contrato negociado socialmente (Rawls, 1971) como método para abordar los dilemas que se presenten durante un estudio. Este contrato procuraría detallar todos los aspectos de un estudio y especificar los dere- 
chos del investigador y de otros participantes. Tanto si existe un contrato formal como si no, siempre existe un contrato informal entre un investigador y las personas que intervienen en un estudio. La conducta ética requiere seguir las obligaciones formales e informales. Este enfoque a las cuestiones éticas se conoce con el nombre de contractualismo y encarna la noción de que los principios morales se generan y reciben su validez mediante los acuerdos a los que llegan personas autónomas, racionales y morales (Kohlberg y Higgins, 1984, p. 483).

Entonces, ¿cómo debemos actuar cuando se plantean dilemas éticos en un estudio? Bell (1989) aboga por que la racionalidad y un sentido de comunidad sean aspectos complementarios de los juicios morales. Con esto quiere decir que las reglas encapsuladas en un código moral junto con atributos humanos como la preocupación por los demás se deberían aplicar conjuntamente. A través de sus acciones, un investigador debería demostrar su adhesión a un código moral mostrando al mismo tiempo preocupación, honestidad y valor para defender los intereses de los demás.

\section{La ética y las culturas gemelas}

Sobre la base de la práctica tradicional en relación a la resolución de dilemas éticos, es evidente que las normas para determinar la acción moral necesitan ir más allá de los contratos formales e informales. Los investigadores deben mostrar un gran nivel de conducta moral exhibiendo preocupación, honestidad y, cuando sea necesario, valentía para actuar de una manera determinada aunque las acciones contempladas puedan no ser populares o agradables.

El investigador tiene que adoptar un código ético de conducta que abarque dos culturas, la del enseñante y los estudiantes por un lado, y la del propio investigador por otro. En ocasiones, los códigos de conducta de las dos culturas dificultan la tarea del investigador. Por ejemplo, la responsabilidad de publicar conocimientos elaborados a partir del estudio de un caso puede entrar en conflicto con la dificultad de garantizar el anonimato de uno de los participantes en el estudio. Si el anonimato de un enseñante no se puede garantizar o si el amor propio de un participante va a quedar perjudicado por la publicación de un informe, no es ético publicarlo. Cuando se informa sobre investigaciones interpretativas es un hecho que siempre hay más cosas que contar que las que realmente se cuentan. Entonces, ¿por qué persistir con un informe específico si resulta perjudicial para un participante en el estudio? Es posible que, más adelante, los datos del estudio se puedan utilizar en informes que sinteticen un programa de investigación. De ser así, es posible que un número suficiente de los factores contextuales carentes de importancia se puedan enmascarar, garantizando así el anonimato de los participantes.

A partir de mi experiencia en investigación en las aulas, ofrezco las siguientes directrices como ejemplos de las precauciones que otros investigadores pueden tener en cuenta para evitar prácticas carentes de ética al realizar investigaciones sobre enseñanza y aprendizaje.

- Antes de empezar un estudio, debemos explicar todos los aspectos del estudio a los participantes. La discusión deberá ser un intercambio sincero en el que se negocien las obligaciones en un contrato informal. El contrato social se puede formalizar en una declaración escrita que represente un consenso sobre las obligaciones de todos los participantes. Si esta declaración se redacta, deberá ser firmada por todas las partes implicadas en la negociación. Así, el contrato se convierte en una base necesaria, pero insuficiente, para resolver ciertos temas que se plantean durante el estudio.

- A menos que los enseñantes estén de acuerdo en que se les cite por su propio nombre, siempre se deben utilizar pseudónimos para la escuela y para los enseñantes que participan en la investigación (es decir, durante todo el estudio y en los informes). Nos hemos encontrado con numerosos problemas 
durante nuestros estudios porque se utilizaban nombres reales en las reuniones de equipo dedicadas a la interpretación de datos. Aunque un enseñante exprese su deseo de ser mencionado por su nombre real, se debe aceptar esta petición con precaución. ¿Qué ocurriría si el enseñante cambia de opinión más adelante? ¿Y qué ocurre con los restantes participantes en el estudio? Probablemente será difícil garantizar su anonimato si una persona es conocida por su nombre verdadero.

- En la medida de lo posible, debemos procurar que los enseñantes participen en las discusiones a medida que se van recopilando datos, que se formulan y estudian cuestiones a investigar y que se hacen interpretaciones.

- Si lo permite el propósito de la investigación, procurar que el enseñante conozca las cuestiones que se están investigando.

- No hablar públicamente de la investigación de una manera que denigre a los enseñantes o a las escuelas.

- Demostrar interés por los enseñantes al ofrecerles feedback sobre enseñanza o aprendizaje y cuando se seleccionen o planteen preguntas para centrar la reflexión. En ocasiones, es mejor no ofrecer feedback y evitar preguntas específicas aunque sean fundamentales para el estudio desde la perspectiva del investigador. La preocupación por el amor propio del enseñante aconseja evitar ciertos tipos de crítica a determinados enseñantes.

- De manera coherente con la preocupación por el amor propio de los participantes, antes de presentar o publicar un estudio debe dejarse que los enseñantes lean cualquier artículo o documento interpretativo preparado a partir del estudio. De ser necesario, se volverán a redactar los escritos teniendo en cuenta los comentarios de los participantes.

- A menos que se pueda garantizar el anonimato de los participantes en un estudio, no se podrá publicar ni presentar nada en un congreso o coloquio sin una autorización por escrito de los participantes.

\section{Conclusiones}

El seguimiento de códigos morales que se aplican a la conducta de la investigación de la enseñanza y el aprendizaje es una condición necesaria pero insuficiente para garantizar una práctica éticamente correcta. Como han ilustrado Sichel (1988), Noddings (1984) y Bell (1989), hacen falta referentes personales como la preocupación por los demás, la honradez y la valentía para potenciar las reglas escritas y las obligaciones contractuales en la resolución de dilemas éticos. Las reglas y los contratos se formulan teniendo en cuenta los intereses de los participantes en una etapa en la que aún no se han planteado dilemas éticos específicos. Cuando se plantea un dilema, las acciones meditadas de un investigador deben tener en cuenta, antes que nada, el bienestar potencial de los participantes en el estudio.

Es evidente que en cualquier estudio habrá innumerables oportunidades para que determinadas acciones se consideren faltas de ética desde la perspectiva de uno o más participantes. A pesar de la magnitud de este problema, no existe ninguna panacea para guiar a los investigadores en todos los contextos y, de hecho, ni siquiera en la mayoría de ellos. En cambio, lo que sí hace falta es preocuparse por el bienestar de los participantes en investigaciones sobre la enseñanza. Una mayor sensibilidad a la naturaleza omnipresente de los aspectos éticos puede ayudar a reducir la incidencia de prácticas poco éticas en la investigación. 


\section{Referencias}

BEL, C. D. Jr. (1989). Justice and caring and the problem of moral relativism. Journal of Moral Education, $18(2), 97-111$.

BERNSTEIN, R. J. (1983). Beyond objectivism and relativism: Science, bermeneutics, and praxis. Philadelphia, PA: University of Pennsylvania Press.

GruNDY, S. (1987). Curriculum: Product or praxis? Londres: Falmer Press.

KOHLBERG, L., y CANDEE, D. (1984). The relationship of moral judgment to moral action. En L. Kohlberg (Ed.), Essays on moral development (Vol. II). The psycbology of moral development: The nature and validity of moral stages. San Francisco, CA: Harper \& Row, pp. 498-581.

KOHLBERG, L., y HIGINS, A. (1984). Continuities and discontinuities in childhood and adult development revisited - again. En L. Kohlberg (Ed.), Essays on moral development (Vol. II). The psychology of moral development: The nature and validity of moral stages. San Francisco, CA: Harper \& Row, pp. 426497.

NoDINGs, N. (1984). Caring: A feminine approach to etbics and moral education. Berkeley, CA: University of California Press.

RaWLS, J. (1971). A theory of justice. Cambridge, MA: Harvard University Press.

SICHEL, B. (1988). Moral education: Character, community, and ideals. Philadelphia, PA: Temple University Press.

TOBIN, K., y CAPIE, W. (1982). Relationships between classtoom process variables and middle school science achievement. Journal of Educational Psychology, 74, 441-454.

TOBIN, K.; ESPINET, M.; BYRD, S. E., y ADAMS, D. (1988). Alternative perspectives of effective science teaching. Science Education, 72 (4), 433-451.

TOBIN, K., y GALLAGHER, J. J. (1987). What happens in high school science classrooms? Journal of Curriculum Studies, 19, 549-560.

TOBIN, K., y JABUNOWSKI, E. (1990). Cognitive processes and teacher change. Ponencia presentada en la asamblea anual de la American Educational Research Association.

TOBIN, K., y ULERICK, S. (1989). An interpretation of bigh school science teaching based on metaphors and beliefs for specific roles. Ponencia presentada en la asamblea anual de la American Educational Research Association.

VON GLASERSFELD, E. (1987). The construction of knowledge. Seaside, CA: The Systems Inquiry Series, Intersystems Publication.

WHEATLEY, G. (1991). Constructivist perspectives on science and mathematics learning. Science Education, $75(1), 9-21$

Aspectos éticos de la investigación en clases de ciencias: dilemas resueltos y no resueltos Kenneth Tobin

CL\&E, 1994, 21, pp. 31-42

Dirección: Kenneth Tobin. Florida State University. Tallahassee, FL 32306.

Artículo original: "Ethical Concerns and Research in Science Classrooms: Resolved and Unresolved Dilemmasa". En Science Education, 1992; 76 (1): 105-117 (1992). Reproducido con autorización de John Wiley \& Sons, Inc. Traducción de Geis Sánchez

(C) PERMISOS PARA CITAR O REPRODUCIR EN OTRAS FUENTES: Se pueden citar libremente hasta 500 palabras. Para reproducir una porción de texto mayor, figuras o ilustraciones, se deberá pedir permiso por escrito a la revista, especificando el uso al que se destina el texto. En todos los casos, se deberá citar el copyright de $C L \& E$. En el caso de artículos o textos que hayan sido a su vez reproducidos en $C L \& E$ los interesados deberán dirigirse tanto a los detentadores del copyright original como a $C L \& E$, en el caso de que se quiera hacer uso de la traducción. FOTOCOPIAS: Para todo lo relacionado con el uso mediante fotocopia del material de esta revista, deberán dirigirse a: CEDRO, C/ José Marañón, 10, $3 .^{\circ}$ Izda. Tel. 5941575 . Fax 4453567 